\title{
Advancing the Science of Developmental Neurotoxicity (DNT): Testing for Better Safety Evaluation
}

Varese, Italy, May 10-13, $2011^{1}$

Anna K. Bal-Price ${ }^{1}$, Sandra Coecke ${ }^{1}$, Lucio Costa ${ }^{2}$, Kevin M. Crofton ${ }^{3}$, Ellen Fritsche ${ }^{4}$, Alan Goldberg ${ }^{5}$, Philippe Grandjean ${ }^{6}$, Pamela J. Lein ${ }^{7}$, Abby Li $^{8}$, Roberto Lucchini ${ }^{9}$, William R. Mundy ${ }^{3}$, Stephanie Padilla ${ }^{3}$, Antonio M. Persico ${ }^{10,11}$, Andrea E. M. Seiler ${ }^{12}$, and Joachim Kreysa

${ }^{1}$ Validation of Alternative Methods Unit, Institute for Health and Consumer Protection, European Commission Joint Research Centre, Ispra (VA), Italy; ${ }^{2}$ Department of Environmental and Occupational Health Sciences, University of Washington, Seattle, WA, USA; ${ }^{3}$ Integrated Systems Toxicology Division, U.S. Environmental Protection Agency, Research Triangle Park, NC, USA; ${ }^{4}$ IUF: Leibniz Research Institute of Environmental Medicine, Duesseldorf and RWTH, Aachen, Germany; ${ }^{5}$ Johns Hopkins University, Center for Alternatives to Animal Testing (CAAT), Baltimore, MD, USA, ${ }^{6}$ Institute of Public Health, Department of Environmental Medicine, University of Southern Denmark, Odense, Denmark; ${ }^{7}$ Center for Children's Environmental Health, University of California, Davis, CA, USA; ${ }^{8}$ Exponent Health Sciences, San Francisco, CA, USA; ${ }^{9}$ Department of Experimental and Applied Medicine, Section of Occupational Health and Industrial Hygiene, University of Brescia, Italy; ${ }^{10}$ University "Campus Bio-Medico", Rome, Italy; ${ }^{11}$ IRCCS "Fondazione Santa Lucia", Rome, Italy; ${ }^{12}$ Federal Institute for Risk Assessment (BfR), ZEBET - Alternative Methods to Animal Experiments, Berlin, Germany

\section{Introduction}

The $3^{\text {rd }}$ International Conference on Alternatives for Developmental Neurotoxicity Testing (DNT3), organized by the European Centre for the Validation of Alternative Methods (ECVAM), Joint Research Centre of the European Commission, was held on May 10 - 13, 2011 in Varese, Italy. Over 100 experts from 18 different countries attended. DNT3 achieved its primary goal of bringing together diverse stakeholders including research scientists, industry representatives, academia, clinical representatives, and experts from regulatory bodies from both Europe and the US. A majority of new participants were from European countries that had not attended the two previous DNT conferences held in the United States in 2006 and 2008. Holding the conference in Europe also facilitated new trans-Atlantic contacts among researchers interested in DNT.

At the regulatory level, there is a recognized need for DNT testing. As early as 1998, the EPA published the Health Effects
Test Guidelines OPPTS 8706300 on DNT (US EPA, 1998) and in 2007 the Organization for Economic Cooperation and Development (OECD) endorsed a new OECD DNT Test Guideline 426 (OECD, 2007). These guidelines are largely based on animal studies and are used as higher tiered, triggered tests based on structure activity relationships or evidence of neurotoxicity in standard adult, developmental, or reproduction studies (Makris et al., 2009).

Experts at the conference stated that these in vivo tests are unsuitable for screening large numbers of chemicals for many reasons including low throughput, high cost, and questions regarding reliability. There was also consensus that new, reliable, and efficient screening and assessment tools are needed for better identification, prioritization, and evaluation of chemicals with the potential to induce developmental neurotoxicity. The information obtained from these screening studies will likely also help to refine animal tests and to inform epidemiological studies.

\footnotetext{
1 This manuscript has been reviewed by the National Health and Environmental Effects Research Laboratory, U.S. Environmental Protection Agency, and approved for publication. Mention of trade names or commercial products does not constitute endorsement or recommendation for use.
} 
The conferees confirmed that DNT is an issue of growing global concern and that damage of the developing brain by chemical exposure results in significant societal as well as individual costs. A particular challenge in DNT is that the neurodevelopmental outcome of exposure to potentially neurotoxic chemicals depends not only on the kind of exposure (dose, duration) but also on the developmental stage at the time of exposure. Participants discussed a variety of alternative methods with this challenge in mind.

There is increasing pressure from a variety of children's health advocacy groups being placed on industry, academia, and regulatory bodies to develop (alternative) methods that reliably and efficiently screen large numbers of chemicals for which we currently have little or no information concerning their potential for developmental neurotoxicity. In vitro and non-mammalian alternative systems-based models are prime candidates, but also in silico methods and combinations should be considered. As a first step, methods are needed for identifying the effects of chemicals on developmental processes critical to the formation of a functional nervous system. Alternative test methods must then be refined to meet regulatory requirements for identifying potential developmental neurotoxicants in a fast, affordable, and reliable way, ideally also providing potency information that is needed for risk assessment. Most likely this can only be achieved with non-animal test methods, but where this cannot (yet) be realized, any new approach should respect animal welfare and the $3 \mathrm{Rs}$, i.e., it should aim to refine, reduce, and ultimately replace animal experiments. Even before regulatory acceptance, scientifically robust methods could be used for prioritizing chemicals for further DNT studies under the EPA and OECD guidelines.
The required features of alternative test methods that would be capable of screening large numbers of chemicals for their DNT profile were discussed in detail. It was proposed that new data must be produced using batteries of in vitro tests that incorporate different DNT-relevant endpoints in high-throughput systems and test a wide range of concentrations of test chemicals. Higher throughput screening methods should be validated if they are used for regulatory purposes.

The applied models must be based on evolutionarily conserved neurodevelopmental processes that are mechanistically relevant for human developmental neurotoxicity. In the next step, these test methods should be validated with the goal of obtaining regulatory acceptance. Moreover, cell-cell and organorgan interactions must be taken into account for valid DNT screening systems, though this requirement presents major technical challenges to non-animal test systems, but is a strength of the non-mammalian alternative animal models.

There was general agreement that methods for a high-throughput screening battery should be developed using recommendations proposed by Crofton et al. (2011), see Box 1. The expectation is that test methods developed with these recommendations would generate reliable data that could be evaluated across test methods and across laboratories and would allow comparison of their predictive capacity and efficiency. This comparative approach would be useful for demonstrating that a given test method performs adequately for the intended purpose (validation) and could hence be accepted for regulatory applications.

Maximizing the opportunities for reducing, refining or replacing animal experiments should be kept in mind during this process. However, participants also agreed that there is still a

Box 1: Examples of questions that need to be addressed in order to develop specific criteria that facilitate comparisons of assays (Crofton et al. 2011)

\section{1) ADVERSITY and SPECIFICITY}

(i.e., to developmental neurotoxicity)

- What is the definition of adversity in your assay or battery of assays?

- How can a specific effect on nervous system development be distinguished from overt toxicity (i.e., cytotoxicity for in vitro tests, death or malformations for alternative species tests)?

- How can the relative potency be compared, within and across assays, for a more complete understanding of the predictive potential of in vivo (i.e., ultimately human health) effects?

- What is the complement of data that will be needed in using "screening and prioritization" assays to support concern (in the regulatory sense) regarding potential developmental neurotoxicity for a given chemical?

\section{2) ASSAY RELIABILITY}

- What type of in silico conditions must be met or defined?

-What is the minimal number of replicates?
- How can variability in the data be expressed?

- What is the minimum number of doses that should be included?

-What dose spacing is optimal (e.g., log, $1 / 2$ log, $1 / 4$ log, etc.)?

- What is the definition of dose?

- How can relative potency be characterized?

\section{3) ASSAY DESIGN}

- How should results be expressed so that there can be comparisons (e.g., NOEC, LD (st.dev), $\mathrm{EC}_{50}, \mathrm{EC}_{30}$, etc.)?

- How do you decide what is a significant difference from control conditions?

- What positive and negative controls should be tested for each type of assay?

- What details regarding specific experimental conditions and methods must be defined?

-What measures of overt toxicity (i.e., cytotoxicity for in vitro tests, death or malformations for the alternative species tests) are appropriate? 
long way to go before we have good models for integrating and interpreting data from in vitro and other alternatives that would be sufficient for assessing human risks.

The conference concluded with views of regulatory officials from the European Commission and the US Environmental Protection Agency (EPA). In their closing remarks, Dr Alan Goldberg (Center for Alternatives to Animal Testing (CAAT, US) and Dr Joachim Kreysa (ECVAM, EU) applauded the progress made since DNT2, but concluded that much work is still needed to develop scientifically robust tests that will provide all stakeholders with the data needed for risk assessment and risk management decisions. Initially identifying those environmental chemicals with the greatest potential to disturb the development of the human brain and central nervous system should receive highest priority.

Dr Goldberg announced that the $4^{\text {th }}$ conference on DNT will be held in the United States and, in recognition of rapid progress in the field, will be scheduled within the next two years.

This report summarizes the main topics discussed during the meeting and presents conclusions and recommendations made for future directions and priorities.

\section{Environmental perspectives of developmental neurotoxicity}

Philippe Grandjean

Since the term 'silent pandemic' was proposed to reflect the prevalence of developmental neurotoxicity in children exposed to environmental chemicals (Grandjean and Landrigan, 2006), the problem has been further characterized and additional substances have been documented as neurotoxicants (Julvez and Grandjean, 2009). A major obstacle, however, remains: convincing 'proof' is required before initiating protection against neurotoxic substances. For several reasons, regulation of developmental neurotoxicants must recognize the known sensitivity of the developing brain. There are many reasons for the use of a precautionary approach to protect the brain functions of the next generation.

First of all, brain development can be highly vulnerable to chemical toxicity, as optimal brain development relies on cell multiplication, differentiation, migration, and cell-cell interactions occurring in a particular sequence and at the right time. Cognition and behavior depend on the functions of the brain as a whole, and optimal function relies on full integrity of the nervous system. Thus, even small deviations from optimal development may lead to dysfunction and risk of disease, with negative consequences for health, quality of life, academic achievement, and economic productivity (Pichery et al., 2011).

In the past, seminal observations were made, but had little immediate consequence, in part because of traditional skepticism toward a new paradigm, and because prevention appeared to be expensive or impractical. Among major discoveries, two pediatricians in Boston discovered that children with lead poisoning did not completely recover, as was thought at the time, but showed mental sequelae and school failure at follow-up (Byers and Lord, 1943). Similarly, early discoveries of methylmercury, ethanol, and polychlorinated biphenyls exposures were not considered sufficient evidence to trigger regulatory action, thus causing delays in preventive actions (Grandjean, 2008a). The expenses caused by these delays in prevention are now becoming clear.

Although the fetus was considered well protected inside the mother's womb, research has shown that the protection may not include environmental toxicants. A study of matched motherinfant samples showed that the placenta offers little protection for most chemicals (Needham et al., 2011), and many environmental chemicals are transferred to the infant via human milk. In relation to body weight, children's contaminant exposures can be excessive. These concerns relate to a large number of substances. Thus, a US study of umbilical cord blood samples showed the presence of 287 environmental chemicals (Environmental Working Group, 2005). The majority of them have not been examined in regard to developmental neurotoxicity.

Evidence of human neurotoxicity from industrial chemicals (other than pharmaceuticals) is based on inadvertent poisonings, occupational exposures, suicidal attempts, and similar observational data, where doses may not be known and control groups are absent. Nonetheless, careful scrutiny of existing data has shown that at least 200 industrial chemicals are associated with neurotoxic changes in humans (Grandjean and Landrigan, 2006). The list of neurotoxicants includes at least 90 pesticides, 25 metals and inorganic compounds, 43 solvents, and 43 other industrial chemicals. These numbers may be a substantial underestimation of the true number of environmental neurotoxicants, but they may reflect a greater neurotoxic risk associated with pesticides and solvents.

With regard to developmental neurotoxicity, all of the 200 adult human neurotoxicants (mentioned above) may be considered capable also of causing damage to the developing brain; however, developmental neurotoxicity has been documented only for an exceedingly small number of these chemicals. Again, such research relies on 'natural experiments' where children have been exposed to a substance pre- and postnatally, and where brain development can be examined in relation to indicators of exposure levels. As many tests are not feasible before school age, or have a low predictive validity in regard to mature brain function, such studies will often require testing of brain functions many years after the prenatal or early postnatal exposure to the suspected neurotoxicant. Thus, research in this field takes a very long time, perhaps a prohibitive duration of follow-up, resources, and commitment. In 1990, a workshop report listed ethanol, PCBs, lead, phenytoin, ionizing radiation, methylmercury, and some drugs of abuse (e.g., cocaine, heroin, methadone) as generally recognized human developmental neurotoxicants (Rees et al., 1990). More recently the evidence of developmental neurotoxicity of environmental chemicals was considered as judged sufficient only for arsenic, lead, methylmercury, polychlorinated biphenyls, and toluene (Grandjean and Landrigan, 2006). Among present day candidates are fluoride, manganese, polyaromatic hydrocarbons, and organophosphate pesticides. This list is likely not to be complete due to the lack of systematic testing for developmental neurotoxicity. 
An additional concern is that exposures to these chemicals during early development can cause brain injury at dose levels much lower than those that affect adult brain (Grandjean and Landrigan, 2006). How low such doses are is only slowly being revealed, e.g., allegedly safe exposures to lead have repeatedly been lowered, and a truly safe level seems to be below exposures that are common today (Grandjean, 2010). This notion therefore puts additional urgency on finding expedient means of identifying developmental neurotoxicants and on preventing hazardous exposures.

A rational strategy will require a multidisciplinary effort. While this conference highlighted the substantial advances made in development and validation of screening test methods, computational chemistry and systems biology should also be utilized. We recently used a large database on protein links and protein-protein interactions to examine the pesticide DDT and its metabolites in regard to their toxicity potentials ( $\mathrm{Au}$ douze and Grandjean, 2011). This in silico approach appeared entirely feasible and useful in pointing to the parent p,p'-DDT substance as the main culprit. Similarly, Willinghagen and colleagues (2011) use computational tools to demonstrate the ability of open sources to predict the effects of ten neurotoxins. Thus, using the whole spectrum of approaches along with clinical and epidemiological evidence, when available, should allow integration of information for use in at least a tentative risk assessment.

Past regulatory action to protect brain development has been, however, impeded by the high level of proof required for decision-making. Interpretation of test results and the rules for decision-making must take into account the individual and societal costs from not acting on information on developmental neurotoxicant exposures. Science has a responsibility to address key issues of importance for protecting developing brains. Traditionally, research has favored skepticism and required extensive replication before acceptance of a hypothesis (Grandjean, 2008b). Although this may be appropriate in many connections, not heeding early warnings on developmental neurotoxicity may ultimately result in adverse effects on the brain functions in today's children and the next generation. The strength of evidence to constitute 'proof' must therefore be seen in a societal perspective, so that the implications of ignoring a developmental neurotoxicant are taken into account. As a further concern, research in toxicology and environmental health has traditionally focused on a small number of chemical substances, while others have been ignored (Grandjean et al., 2011). The well-known chemicals that have been most extensively researched include developmental neurotoxicants, such as arsenic, lead, mercury, and polychlorinated biphenyls.

There is limited evidence for the neurotoxicity in humans (adults) of over 200 chemicals (Grandjean and Landrigan, 2006) and 100 or more developmental neurotoxiciants (Crofton et al., 2011), but there is a complete absence of testing of thousands of routinely used commercial chemicals. This lack of data is a huge uncertainty in the need to protect human health, especially the developing nervous system.

\section{Clinical perspectives of developmental neurotoxicity}

Roberto Lucchini and Antonio Persico

During the conference it was acknowledged that the human population displays large genetically-driven inter-individual differences in sensitivity to potential DNT agents. This variability spurs interest into designing a feedback validation process, whereby the same in vitro test batteries used to screen chemicals can later be also applied to cellular models derived from specific patient populations to test for possible individual hyper/hyposensitivity to those chemicals. In particular, two paradigms were presented, whereby available evidence supports a significant interaction between predisposing genetic variants and exposure to developmental neurotoxicants: (a) the cumulative combined exposure to pesticides and metals, and the presence of specific genetic variants shown to interact in determining the risk threshold for neurodegenerative disorders, such as Parkinson's Disease, Parkinsonism and Parkinsonian disturbances in general; (b) a prenatal exposure to specific DNT agents in conjunction with a vulnerable genetic background, as implicated in the pathogenesis of autistic disorder by specific gene-environment interaction models.

Parkinson's Disease (PD) is a neurodegenerative disorder that typically affects individuals in their old age, after loss of dopaminergic neurons in the nigrostriatal portion of the brain. The number of these cells declines with age at a rate of 5-10\% per decade and the characteristic symptoms develop once about $70 \%$ of the dopaminergic neurons in the substantia nigra have been destroyed (Nadeau, 1997). The incidence of the disease is increasing worldwide, given the increasing life-expectancy and duration of individuals' working lives. Recent estimates predict an astonishing increase of neurodegenerative diseases in subsequent years not only for elderly people, but also within the middle aged population (Albert, 2007). Therefore this trend may not only relate to the increasingly aging population per se, but also to a wider and more frequent exposure to neurotoxicants. This growing trend is estimated to affect mostly those countries that are now rapidly industrializing which have poorly controlled exposure conditions, such as China, India, Brazil, and Bangladesh (Dorsey et al., 2007).

An important contribution to these diseases may be inadequate control of lifetime exposure to neurotoxicants, starting from prenatal life to old age. Early life exposure is likely to play a key role within the lifetime exposure scenario, given the potential increased susceptibility to further occupational or environmental or dietary exposure occurring in adult life. Pesticides and heavy metals are being related by human epidemiological studies and animal data to PD and, more in general, to Parkinsonian disturbances. Differences in epidemiological classification distinguish "classical" idiopathic PD from Parkinsonism (Elbaz et al., 2002), and environmental exposure is considered a potential etiological factor of Parkinsonism, especially for individuals with a genetic predisposition. Polymorphisms in the promoter region of the $\alpha$-synuclein gene confer a higher susceptibility to idiopathic PD. Thus, interactions with exposure to neurotoxicants could play a relevant role in human Parkinsonism (Vance et al., 2010). 
Manganese is a neurotoxicant potentially implicated as an environmental factor in the pathogenesis of Parkinsonism. The prevalence of Parkinsonian disturbances has been found to be significantly higher among lifetime residents in the vicinities of ferroalloy plants in Italy and is significantly correlated with the manganese level in the deposited dust (Lucchini et al., 2007). Similarly, increased prevalence of PD was observed in relation to the concentration of manganese in total suspended particles in Canada (Finkelstein and Jerrett, 2007).

Important evidence from developmental models in mice has shown that prenatal or perinatal exposure to a range of environmental chemicals, including paraquat, maneb, and organochlorine pesticides, may either directly cause a reduction in the number of dopaminergic neurons in the substantia nigra, or cause an increased susceptibility to degeneration of these neurons with subsequent environmental insults or with aging alone (Cory-Slechta et al., 2005). Based on the importance of early life exposure within the lifetime exposure model, DNT testing must be able to predict the long term neurodegenerative effects caused by substances acting with a cumulative mechanism of toxicity.

Autism Spectrum Disorder (ASD) is a neuropsychiatric disorder characterized by deficits in social interaction and communication, as well as by stereotypic behaviors and insistence on sameness, with onset in early childhood prior to three years of age. Its incidence has dramatically risen during the last two decades from 2-5 per 10,000 to approximately $20-60$ per 10,000 children. Broader diagnostic criteria and increased awareness in the medical community have contributed to this trend, but a real increase in incidence possibly due to gene-environment interactions is also likely (Grether et al., 2009; Hertz-Picciotto et al., 2006; King and Bearman, 2009).

Microscopic neurodevelopmental abnormalities uncovered in ASD brains strongly support a prenatal origin for autism (Bauman and Kemper, 2005), although early postnatal exposures could also modulate the behavioral phenotype, as may occur with p-cresol (Altieri et al., 2011). Genetics is believed to contribute strongly to the pathogenesis of ASD, but initial heritability estimates above $90 \%$ (Persico and Bourgeron, 2006) have not been confirmed by more recent twin studies, which support a larger proportion of variance explained by shared environmental factors as opposed to genetic heritability ( $55 \%$ vs $37 \%$ for strict autism, respectively) (Hallmayer et al., 2011). Furthermore, many patients reveal no causal mutations or copy number variants.

This unexpected complexity has spurred interest into polygenic models allowing for gene-environment interactions to produce large inter-individual phenotypic variability. The validity of such models is also supported by known prenatal teratological agents, such as rubella or cytomegalovirus infection, and drugs like thalidomide, and valproic acid, which yield autism only in a subset of presumably vulnerable individuals (Landrigan, 2010).

Specifically, three gene-environment interaction models have been presented:

1. RELN-PON1-prenatal exposure to organophosphate pesticides or excessive oxidative stress (Persico and Bourgeron, 2006): the RELN gene encodes for Reelin, a stop signal critical to neuronal migration in several regions of the central nervous system including neocortex, cerebellum, and hindbrain. The PON1 gene encodes for an enzyme usually defined as "paraoxonase," which exerts several enzymatic activities including the inactivation of some organophosphorus compounds, the degradation of lipid peroxides preventing atherosclerosis and vascular disease, the breakdown of bacterial endotoxins, and the prevention of protein lactonation. Autistic individuals display a significant reduction in Reelin protein levels, as well as reduced serum PON1 enzymatic activity (Gaita et al., 2010). RELN gene polymorphisms yielding lower Reelin gene expression both in vitro and in vivo, are significantly associated with autism, as occurs with PON1 gene variants responsible for lower gene expression and reduced organophosphate detoxification. Therefore, individuals carrying genetic variants producing lower amounts of Reelin and PON1 enzyme, if exposed to developmental neurotoxicants like organophosphorus pesticides during critical periods in prenatal neurodevelopment, could be especially vulnerable to undergo abnormal neuronal migration yielding altered neural networks and behavioral autism (Persico and Bourgeron, 2006). This model has recently received experimental and epidemiological support both in humans and in mouse models (Eskenazi et al., 2010). An alternative mechanism to explain this interaction places greater emphasis on excessive prenatal and early postnatal oxidative stress, partly OP-driven, than on cholinesterase inhibition (Gaita et al., 2010).

2. ATP2B2-SLC25A12-prenatal exposure to polychlorinated biphenyls (PCBS): excessive intracellular $\mathrm{Ca}^{2+}$ has been shown to play a relevant role in the pathophysiology of autism (Krey and Dolmetsch, 2007). The ATP2B2 gene encodes for the plasma membrane calcium ATPase 2 (PMCA2), which removes $\mathrm{Ca}^{2+}$ from the cytoplasm into the extracellular space. It is especially critical to the function and plasticity of critical synaptic sites, such as the parallel fiber-to-Purkinje cell synapse in the cerebellar cortex and in parvalbumin-positive GABAergic interneurons. ATP2B2 gene variants, possibly yielding reduced gene expression and slower $\mathrm{Ca} 2+$ clearance, are associated with autism in males (Carayol et al., 2011). Excessive intracellular $\mathrm{Ca}^{2+}$ spikes can in turn interfere both with neurodevelopment and with energy production, modulating the activity of the mitochondrial aspartate-glutamate carrier AGC1. Interestingly, also the SLC25A12 gene, which encodes for AGC1, encompasses genetic variants able to influence disease risk in autism (Palmieri et al., 2010). Genetic vulnerability at the ATP $2 B 2$ and SLC25A12 loci could be exacerbated by prenatal environmental exposure to $P C B S$, important endocrine disruptors also able to promote $\mathrm{Ca}^{2+}$ entry from the extracellular space, and $\mathrm{Ca}^{2+}$ release from the endoplasmic reticulum (Pessah et al., 2010).

3. MET-prenatal exposure to polycyclic aromatic hydrocarbons (PAHs): the MET protooncogene encodes for the MET receptor tyrosine kinase, a key modulator of cell proliferation and migration, as well as neurite outgrowth and synaptogenesis in the development of the CNS, gut and immune system (Campbell et al., 2006). ASD brains display profound 50\% decreases in MET mRNA and protein on average, compared 
to matched controls; the MET gene promoter contains the single nucleotide polymorphism (SNP) rs1858830, whose C allele is significantly associated with autism and with lower MET transcription both in vitro and in post-mortem brains (Campbell et al., 2006, 2007). Prenatal exposure of mice to the PAH benzo(a)pyrene blunts postnatal MET gene expression and yields behavioral deficits in a novelty test (Sheng et al., 2010). Therefore, prenatal exposure to PAHs may either act in conjunction with the C allele at SNP rs 1858830 further enhancing autism risk, or alternatively may reduce the protection conferred by the $G$ allele.

These three examples of gene-environment interaction are based on growing genetic, neurobiological, and epidemiological evidence, not merely on theoretical grounds. They are in no way exhaustive, as prenatal exposure to many other chemicals could favor the development of autism by interfering with the intracellular pathways best shown to underlie this disease (excessive $\mathrm{Ca}^{2+}$ signaling, PI3K/mTOR/ERK, oxidative stress, decreased synaptogenesis, epigenetic abnormalities, decreased oxidative phosphorylation). These models merely exemplify the need to consider the encounter between DNT agents and an individual, or even a fetal-maternal unit. In addition they indicate that whatever battery of alternative developmental neurotoxicity test will eventually be defined, it should also be employed to assess DNT risk in selected patient populations and their first-degree relatives, who may have a lower sensitivity threshold for the negative consequences of environmental toxins as compared to the general population.

\section{State of science: in vitro DNT models and endpoints}

Ellen Fritsche

Pharmaceuticals and industrial chemicals are known to adversely impact neurodevelopmental processes critical for normal brain development, including: neural progenitor cell (NPC) proliferation, migration, differentiation into neurons and glia, apoptosis, myelination, synaptogenesis, network formation, as well as general or neuronal subtype-specific cytotoxicity (seen in vivo as necrosis) or gliosis. In vitro systems cannot mimic the complexity of in vivo brain development; however, it is now possible to investigate effects of compounds on these processes which, when disturbed during critical phases of development, result in the formation of a suboptimal organ.

In vitro models which mimic one or more of these endpoints are numerous and most current models cover three common species: mouse, rat, and human (Coecke et al., 2007; Bal-Price et al., 2010). Rat cells are commonly used as a cell line or as primary cultures. One of the most extensively studied is the PC12 cell, a rat pheochromocytoma cell line which has been used since 1976 (Greene and Tischler, 1976). Primary cultures of rat cerebellar granule cells are widely used in in vitro models. Gene expression analysis has been postulated to be a useful tool for predicting DNT in such cultures (Hogberg et al., 2009; Hogberg et al., 2010). From the mouse, several neuroblastoma cell lines are available, e.g., N2a cells (Amano et al., 1972) or N1E-115 cells
(Richelson, 1973). In addition to tumor-derived lines, multiple mouse stem cell-derived clones are available, including mESD3 cells. This cell line is the basis for the validated embryonic stem cell test where the cells differentiate into beating cardiomyocytes (EST; Spielmann et al., 1997; Seiler and Spielmann 2011). The mES-D3 cells are also utilized for neural differentiation and show promise for use in screening chemicals for DNT (Seiler et al., 2007). Mouse neural progenitor cells (mNPCs) can be prepared from embryonic or postnatal mouse pup brains of different ages. Such progenitors grow as neurospheres, develop in vitro, and are especially valuable as a source of genetically manipulated primary cells (Gassmann et al., 2010).

Human cell systems are recommended as the most relevant in the context of the $21^{\text {st }}$ century approach in toxicity testing: getting a better prediction of human toxicity by using human in vitro models (NRC 2007). Numerous human models are currently available, ranging from neuroblastoma lines to stem cell-derived systems (reviewed in Coecke et al., 2007). Multiple human neuroblastoma cell lines like SH-SY5Y or IMR-32 cells (e.g., Kohl et al., 1980; Reynolds and Pérez-Polo, 1975) have been applied to many different research questions. However, they have a disadvantage in that the expression profiles in these cells contain many tumor growth genes. For example, the MEIS homeobox genes are overexpressed in neuroblastoma cells and this gene amplification influences downstream targets involved in developmental signaling pathways, chromatin binding, cell cycle control, proliferation, and apoptosis (Geerts et al., 2003). Moreover, their ability to respond to oxidative stress is impaired (Moors et al., 2009).

LUHMES (Scholz et al., 2011) and ReNcell CX (Donato et al., 2007; Breier et al., 2008) cells are not tumor-derived, but instead are immortalized cells of primary origin (e.g., by c-myc overexpression). This also limits their usage in predicting the effects of chemicals on neural cell proliferation, but might allow the study of some other endpoints. Alternative human in vitro systems like progenitor- or stem cell-based methods have been studied intensively over the past decade because they are primary, of human origin, and expandable although not immortalized (reviewed in Breier et al., 2009). Another advantage of some primary cell cultures is the simultaneous differentiation into the physiological cell types of the brain, namely neurons and glia cells (Moors et al., 2007 and 2009). Presence of glia is well known to affect toxicity of chemicals towards neurons. Therefore, a mixed, more organotypic culture is preferred for chemical testing.

A number of specific cell based methods have been subject to more in-depth investigations for suitability in toxicity testing. These include stem/progenitor cells derived (i) from human umbilical cord blood stem cells, (ii) directly from human fetal brain tissue, or (iii) from human embryonic stem cells (ESC). The earliest work on such systems included (i) a neural stem cell line generated from human umbilical cord blood stem cells (HUCBNSC line; Buzanska et al., 2005) and (ii) human neural progenitor cells (NPCs) growing as neurospheres (Fritsche et al., 2005). The difference in time of origin of these cells (embryonic vs. fetal) is complementary, covering the embryonic and early fetal period of neurodevelopment, respectively. Both methods 
have been further developed and characterized, especially for their DNT testing applicability ((i): Sun et al., 2005; Jurga et al., 2006; Buzanska et al., 2009; Zychowicz et al., 2011; (ii): Moors et al., 2007, 2009; Schreiber et al., 2010; Gassmann et al., 2010; Tegenge et al., 2010; Fritsche et al., 2011). Human embryonic stem cells were recently differentiated in vitro for the purpose of DNT testing. Ylä-Outinen et al., (2010) differentiated ESCderived NPCs on multi electrode arrays (MEAs) and observed impaired electrical activity in the presence of methylmercury. Further development and validation of such techniques to allow medium throughput testing is desired.

All these different cell models from various species raise the question on the predictivity of the individual models for human DNT. No comprehensive investigation on this topic has been carried out so far; however, this issue among others is currently investigated in the German BMBF (German Federal Ministry of Education and Research) joint project on the development of predictive in vitro tests for DNT (Seiler et al., 2007). There are indications that sensitivity towards chemicals (endpoint: viability) can be similar within tumor cells across different species, e.g., rat pheochromocytoma (PC12), mouse neuroblastoma (N1E-115), and human neuroblastoma (SH-SY5Y) cells (Mundy et al., 2010). In contrast, normal and tumor cells of the same species can differ in their sensitivity towards compounds (Radio et al., 2010; Harrill et al., 2011a). Nevertheless, tumor-derived cells might still be useful for certain applications (Sarkanen et al., 2007; Gustafsson et al., 2010; Podrygajlo et al., 2010). Species differences of normal, non-tumor cells, however, are an understudied field of research. Besides sporadic publications (Abbott et al., 1999; Gassmann et al., 2010) there are very few data on direct species comparisons with regard to neurodevelopmental toxicity pathways. It is necessary to understand species differences in the biology of neurodevelopmental signaling pathways and resulting toxicity of chemicals.

Which endpoints have been studied in which models so far? The most thoroughly investigated endpoints to date are neural cell proliferation and neurite outgrowth. The US EPA has generated a large amount of data from lots of chemicals across different cell types and species for these two endpoints (Radio et al., 2008; Breier et al., 2008; Mundy et al., 2010; Radio et al., 2010; Harrill et al., 2011; Harrill et al., 2011a; Robinette et al., 2011). For chemical effects on neural differentiation, migration, synaptogenesis and functional network activity, less data is available. Differentiation of HUCB-NSC, NPC, or rat CGC into neurons and/or oligodendrocytes have also been assessed using more limited numbers of chemicals (Buzanska et al., 2005; Hogberg et al., 2009; Fritsche et al., 2005; Moors et al., 2009, Schreiber et al., 2010). Not only the ability to differentiate, but also to reach the correct position by cell migration can be studied, preferably in spherical models (Moors et al., 2007; Moors et al., 2009; Schreiber et al., 2010; Tegenge et al., 2010). Besides these basic cell biological endpoints, chemical effects on more functional aspects of neuronal cells have been investigated recently in different cell models, mainly synaptogenesis (Mundy et al., 2008; Podrygajlo et al., 2010, Scholz et al., 2011, Harrill et al., 2011) and network activity (Görtz et al., 2004; Jurga et al., 2009; Frimat et al., 2010; Ylä-Outinen et al., 2010; Scholz et al.,
2011; Novellino et al., 2011; Hogberg et al., 2011).

It is clear that methods and models are now available that cover most of the processes important for neurodevelopment. These include NPC proliferation via migration, differentiation, neurite outgrowth to synaptogenesis, and network formation. Such individual tests could possibly form a DNT testing battery in the future. Most of the endpoints are currently low-throughput and a critical need for future research is to scale-up these models to medium (e.g., hundreds of chemicals per month) or high throughput (e.g., thousands of chemicals per week). What we don't know yet among the multiple cell models and the distinct endpoints is which models are the most predictive. More than likely a certain combination of endpoints and models will be needed to obtain the information necessary to predict in vivo DNT. Computational modeling and generation of data from chemical test sets of DNT positive and negative compounds across a variety of the most promising models and endpoints is needed.

Research on alternative in vitro methods and models for DNT testing has at least three major data gaps to fill: (i) systematic comparison of tumor/non-tumor cells, (ii) identification of species differences and similarities, and (iii) identification of DNT relevant pathways which contribute to DNT and/or determine cell type and/or species differences. Over all, in vitro testing for DNT follows the same path as the rest of the 'alternatives to animal testing' field is moving: using human, three dimensional, cellular systems for testing disturbances of pathways relevant to human, cell-specific, physiological cellular functions.

\section{Potential of non-mammalian species as relevant DNT models}

Stephanie Padilla

Studies of the development of "lower", alternative species have revealed that the mechanisms underlying the development and function of the nervous system are surprisingly conserved across the phylogenic tree. That is, many of the basic molecular developmental events are identical in mammals and in the alternative species. Within the last 10 years, these alternative species (e.g., small fish models such as the zebrafish Danio rerio) have gained popularity as vertebrate models for screening for developmentally toxic, specifically neurotoxic, chemicals (see a recent review by Padilla and MacPhail, 2011). The popularity of alternative species is due primarily to three aspects: (1) molecular biology has revealed the basic concordance of cellular events in a wide range of small fish species to that in mammalian species, including humans; (2) the concordance has been verified with advances in genetics and pathway analyses (as a result, these alternative species are now being used increasingly in probing the basic processes of life and disease); and (3) the size and speed of development of small fish makes their use particularly ideal for high throughput assays.

Zebrafish represent an attractive complementary species for developmental neurotoxicity assessments. Some of the major hurdles associated with in vitro screening are minimized when testing with an intact organism. Because the zebrafish liver ma- 
tures early, the embryo is able to metabolize many protoxicants into the active metabolite(s) (Tao and Peng, 2009; Goldstone et al., 2010). Nervous system development is three dimensional with non-neuronal cells playing major roles as they do in the development of the mammalian nervous system. Moreover, as in the mammalian nervous system, both central and peripheral nervous system are present and protected by blood/nerve and blood brain barriers. For a clear, concise review of the early development of the zebrafish nervous system, see Guo (2009). Unlike mammalian experimental models of neurodevelopment, it is extremely easy to modify development through use of morpholino knockdowns (for a review see Bill et al., 2009) and additionally, to create fluorescent "reporter" fish for ease of assessment (e.g., Higashijima, 2008; Tsang, 2010). One of the most important aspects that a small fish model brings to any screening effort is the ability to assess the integration of nervous system development via behavioral assessments. Because only six days of development are required for a zebrafish larva to display sensory, motor and cognitive functions, in less than a week an investigator can determine whether developmental chemical exposure altered nervous system development.

Many of these advantages were exploited by investigators presenting at the DNT3 meeting. It was shown that behavioral alterations, whether basal or stimulus-controlled locomotor activity, habituation, spontaneous movements (i.e., tail coiling) or reflex movements (i.e., startle response) in larval zebrafish after developmental exposure often were able to distinguish between neurotoxic and non-neurotoxic chemicals. Work in multiple laboratories indicated that behavioral endpoints in zebrafish larvae are advantageous endpoints for predicting developmental neurotoxicity because the tests were able to distinguish between compounds known to be developmentally neurotoxic and chemicals known to be negative for developmental neurotoxicity. Morphometric endpoints were also explored with some groups presenting medium-throughput morphometric assessments of exposure to neurotoxic compounds using whole-mount immunostaining with neuron specific antibodies or in vivo imaging of neuronal cells using novel fluorescent dyes. Combination of morphometric and behavioral endpoints in developmentally treated fish allows the researcher to capitalize on the positive aspects of the zebrafish model while still being able to attain a low to medium rate of throughput.

In comparison to the alternative animal model work presented at the DNT2 meeting, the researchers at the DNT3 meeting tended to present data comparing the developmental toxicity of more extensive panels of test chemicals, and also emphasized developing higher throughput methods for all endpoints.

\section{Application of alternative approaches for regulatory purposes}

Abby Li

There are several potential applications of alternative approaches for regulatory purposes, including: (1) providing mechanistic data for a specific chemical to better understand effects observed in whole animals, including extrapolation between rodents and humans;(2) grouping of chemicals based on similar mode of action (MOA); (3) supporting decisions for regulatory actions currently based primarily on structure activity relationship; (4) screening chemicals for further in vivo testing; and (5) replacing in vivo testing for regulatory decision-making. The level of validation required for these purposes will depend on the defined use of the alternative approaches. Significantly more validation is needed before application of these approaches can be used for screening or replacing in vivo DNT testing.

The use of alternative approaches for the first two applications can be achieved immediately, with proper validation of the specific assay system(s) to test hypotheses regarding mode of action (MOA) for a particular chemical or defined group of chemicals. Clear descriptions of each of the key events (i.e., measurable parameters) linking external dose to biological perturbation to ultimate toxicological effect of concern are required to support an MOA (Seed et al., 2005). Data based on integration of animal or human in vitro or in vivo data including information on toxicity pathways can be used to improve understanding of MOAs and focus further testing and risk assessment decisions on the most sensitive endpoints of concern (Plunkett et al., (2010). In addition, alternative methods can be used to aid in grouping chemicals based on similar mode of action which can: (1) support decisions on cumulative risk assessment involving exposures to multiple chemicals with the same mode of action, and (2) reduce animal testing by supporting use of toxicology data for a representative chemical for a group of related chemicals with similar mode of action (GAO, 2005; US EPA, 1999, 2010a). In cases where regulatory decisions are based solely on structure activity due to the lack of any available toxicity data, data from alternative nonmammalian or in vitro approaches may provide additional data to inform regulatory decisions (GAO, 2005; US EPA 2010a). However, recognition of the limitations of these data should be clearly acknowledged.

The focus for DNT3 has been on the development and use of alternative methods for screening chemicals for developmental neurotoxicity. The science is at an early stage of development and significant efforts are needed to validate a battery of tests based on guidance from Crofton et al. (2011). Crofton et al. (2011) provides general principles and recommendations to "facilitate development of alternative methods for screening substances for potential developmental neurotoxicity", and primarily focuses on protocol optimization and standardization so that methods can be compared across laboratories. Reliability and repeatability under large-scale conditions (standardization and economy of scale) is needed, which is very different from small scale use of these technologies to address specific research questions. In order to accomplish this, the general guidance from Crofton et al. (2011) needs to be translated into specific recommendations appropriate for each type of assay.

Towards this effort, questions were developed by regulatory and industry scientists participating on the last day of the session that identify important issues that need to be addressed in order to evaluate data from alternative methods for use in screening chemicals for further testing (Fig. 1). Important issues to address include definition of an adverse or biologically 


\title{
An Integrated Evaluation Strategy for the Development of New Test Methods and Use of Data
}

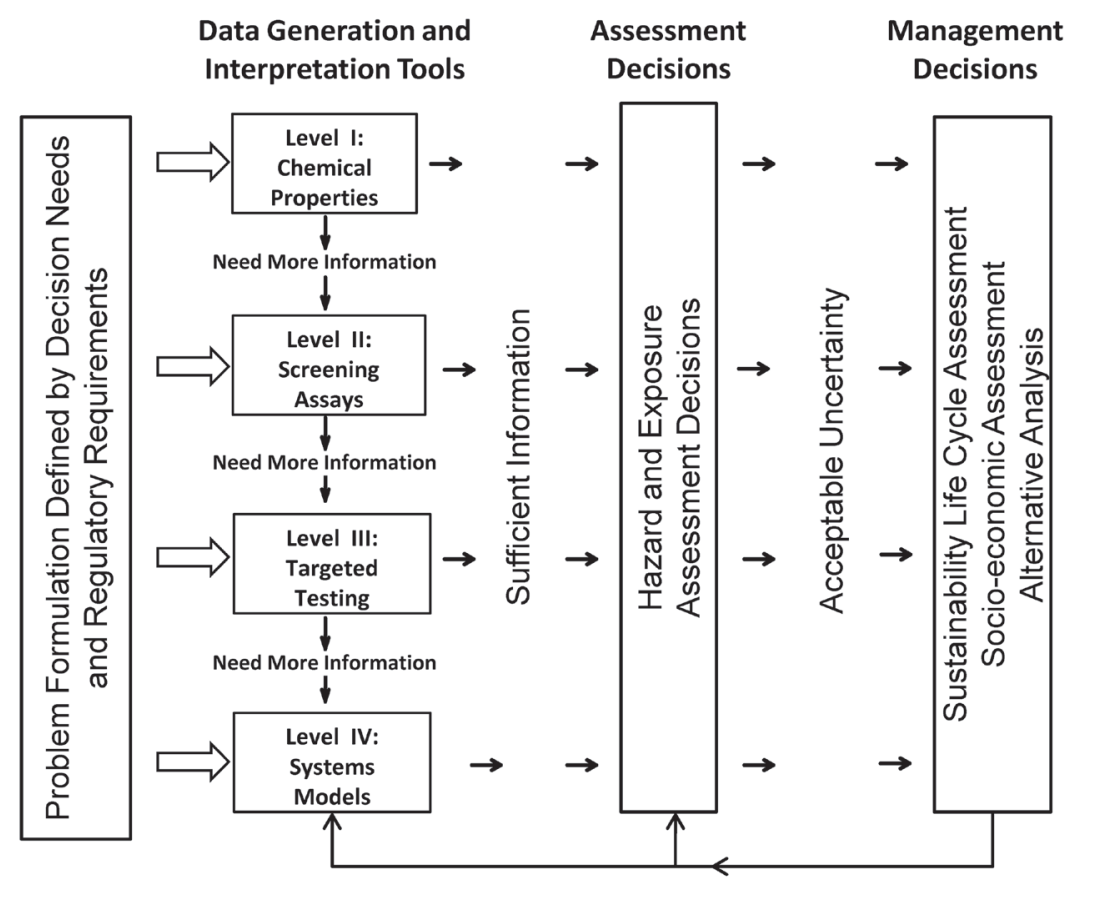

\author{
Information
Needs \& \\ Complexity Uncertainty
}

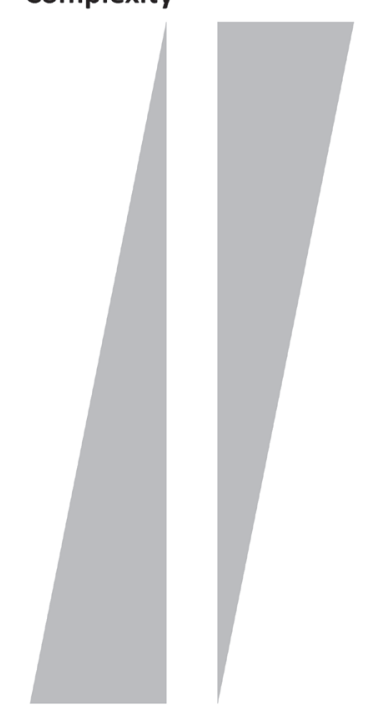

Fig. 1: An Integrated Evaluation Strategy for the development of new testing methods and the use of data in assessment and management decisions.

The relationship between testing, assessment, and management research for chemicals is driven by problem formulation as defined by decision-making needs. In this tiered evaluation strategy the amount of resource utilization is adjusted for each level to provide efficient and timely data to address the needs of particular decisions. New methods need to be developed at all four levels of data generation to allow for the most efficient assessment of the potential hazards of chemicals to the developing nervous system (adapted from US EPA, 2011).

significant effect for the specific assay, minimum number of dose levels tested (e.g., 10) and replicates (e.g., 3), reporting of data (e.g., $\mathrm{IC}_{50}$ and standard deviations), standardization of dose-response comparisons with cytotoxicity or overt toxicity, detailed and transparent reporting of specific experimental conditions, and development of a common training set of positive and negative controls (e.g., 5 positive and 5 negative controls including chemicals with toxicity in other systems, but not developmental neurotoxicity) for different types of assays (Fig.1). Currently, comparisons across laboratories and different assays are hampered because of the lack of specific criteria. These criteria should also be basic inclusion criteria for datamining computational efforts to link results from alternative methods with animal or human outcomes similar to those initiated by Martin et al. (2011) for developmental outcomes

In vitro and alternative non-mammalian models are promising approaches for screening and prioritizing chemicals. In order to focus toxicity testing on the most sensitive adverse effects, it will be necessary to provide concentration-effect or dose-response data that are comparable with data that are being developed for other toxicity endpoints. Ensuring that data generated today are of the highest quality will lay a stronger foundation for reaching the ultimate goal of rapid and streamlined review and assessment to ensure the availability of safer and more effective products for the consumer, at reduced costs and environmental and health impacts.

\section{Conference conclusions and recommendations}

The conference achieved its objective of bringing together the stakeholders and experts from diverse scientific disciplines and representatives from relevant industries to discuss alternative tools for assessing the potential of chemicals to cause developmental neurotoxicity. Since DNT3 was the first of this series of meetings to be held in Europe, many more European experts 
and stakeholders participated and joined the discussion on DNT alternatives, bringing to the table their own data and experience. At the conclusion of the conference, general consensus was reached on the following:

1. There is an urgent need to develop alternative test systems/ testing strategies that will allow for faster and more cost efficient testing of chemicals for developmental neurotoxicity. Critical to these methods is the need to predict adverse neurodevelopmental outcomes in humans.

2. DNT models with high throughput capacity are needed to deal with the very large number of chemicals that have to be tested for DNT potential.

3. There has been significant progress since the last DNT2 meeting in applying in vitro and non-mammalian test systems to DNT, however, much remains to be done to validate reliable alternative test systems for identifying chemicals with DN potential.

4. While there is general consensus that a battery of alternatives will be needed, at present there is insufficient data to determine which models and/or which endpoints are minimally required to reliably screen for human DNT potential.

5. A critical need is to generate data with each alternative method using a large number of test chemicals across a wide range of concentrations. Preferably a comparable or similar list of test chemicals should be used in each model and in each laboratory to facilitate comparison of the results.

6. It is necessary to identify a reference set of chemicals for evaluating the reliability and relevance of alternative DNT models. This includes both in vitro models and alternative species.

7. Cell-based assays mimicking important key aspects of brain development (proliferation, migration, differentiation, synaptogenesis, network formation, etc.) should be applied as in vitro DNT functional endpoints for use in drug discovery, hazard identification, and risk assessment.

7. While available in vitro models cannot mimic the complex interactions triggered by an initial chemical challenge to neuronal damage, they may have the capacity to assess whether a chemical triggers critical mode(s) of action (MoAs). Their importance will therefore increase once these critical MoAs are identified and suitable tests developed.

8. There is a need for data comparing similar endpoints in models from different species (human versus rat versus mouse versus zebra fish, etc.).

9. Computational toxicology and modeling should also be applied to evaluate the predictive capacity of tests and test batteries.

10. Relevance of the data from in vitro and alternative species has to be cautiously evaluated when extrapolating to humans.

11. Identified pathways of toxicity specific for DNT should be used to develop new endpoints and assays.

12. The future success of applying these approaches for risk assessment purposes or data-mining computational efforts depends upon the quality and reliability of the data generated today. Specific criteria based on general principles outlined by Crofton et al. (2011) are needed so that proto- cols can be optimized and standardized for cross-laboratory validation.

13. The majority of conferees agreed that more "hard facts", indicators, and evidence are needed to make risk assessment decisions. However, others expressed concern that the protection of the public is not necessarily improved by premature decisions. With proper validation, alternative approaches can be used to screen chemicals and accelerate collection of evidence on higher priority chemicals. As pathways of toxicity specific for DNT are identified, they should be used to develop new endpoints and assays.

14. A plea was made to apply the precautionary principle, which would require to introduce preventive actions already before final proof is available, but the majority of conferees agreed that more "hard facts", indicators, and evidence are needed. The modern tools, i.e. alternative approaches discussed at the conference, should accelerate the collection of such evidence.

\section{The future of DNT: an integrated evaluation strategy for the development and use of DNT data}

Kevin Crofton and William Mundy

As this report demonstrates, substantial progress has been made in the development of alternative test methods for DNT: 1) In vitro and alternative species models have been developed based on the conserved, key events of neurodevelopment, 2) assay protocols have been evaluated for their application in chemical testing using the guidance provided in Crofton et al. (2011), and 3) data is now being generated using a common test set of chemicals in order to determine the predictive ability of various assays and their possible use in a tiered approach to chemical screening and prioritization (Radio et al., 2008; Breier al., 2008; Harrill, 2011; Shafer, 2011). However, as described by $\mathrm{Li}$ (Chapter 6) there is a need to use the data generated in these assays for regulatory purposes beyond chemical prioritization. Figure 1 illustrates an Integrated Evaluation Strategy which is a general framework for the generation and use of data at multiple levels in making regulatory decisions (US EPA, 2011). The overall goal is efficiency: develop only the data needed for making regulatory decisions using the least amount of resources possible. The amount of resource utilization is adjusted for each level to provide efficient and timely data to address the needs of particular risk decisions.

This Integrated Evaluation Strategy envisions different levels of information needs/generation (illustrated by the four levels in Fig. 1), with each level characterized by increasing complexity and cost. The bars to the far right of the figure show how resource needs increase with needs for reduced scientific uncertainty in estimates of hazard and risk. The evaluation strategy ranges from an initial risk assessment that requires few resources to evaluate large numbers of chemicals (while tolerating high levels of uncertainty) to a more complex, advanced risk assessment that requires substantial resources to evaluate only a few chemicals (with lower levels of uncertainty). Although shown 
as discrete levels, the sequence actually represents a continuous process that proceeds until sufficient information to support a particular decision has been obtained.

The development of information at Level 1 uses an understanding of the inherent properties of chemicals for use in QSAR and/ or Read Across based assessments. Currently there are no accepted QSAR models for DNT. New QSAR models for DNT are needed and will require data from the testing of large numbers of chemicals, data not currently available. The in vitro approaches outlined by Fritsche (Chapter 4) will be useful in Level 2 assessments. These methods are much more cost-effective compared to in vivo testing and allow for higher throughput testing. However, decisions based solely on data from these methods will involve a high level of uncertainty, including extrapolation of from in vitro to in vivo (e.g., Andersen and Krewski, 2009). Uncertainties are large when extrapolating from methods that may rely on isolated protein preparations or clonal cell lines. Data generated from these cost efficient in vitro methods will allow population of Level 1 QSAR models, as well as improve Read Across efforts. In addition, these Level 2 methods can be used to test and refine QSAR and Read Across models used in Level 1.

Outputs from the upper two levels are currently being proposed for use to prioritize chemicals for further testing (see, for example, Reif et al., 2010). The goal of Level 1 and 2 is to test large numbers of chemicals, and hypothetically prioritize those chemicals for further testing. This prioritization should include a narrowed list of possible adverse outcomes (e.g., cancer or reproductive toxicity) that will replace the current regulatory requirement to test every possible target. Recent examples of approaches to this include the development of toxicity 'signatures', or patterns of in vitro outcomes that are associated with significant probabilities for specific adverse outcomes (Martin et a., 2011; Judson et al., 2011). While this is a logical and cost efficient step-wise process there are major challenges in this approach. The first is building the scientific database to assure confidence that this narrowed focus does not result in unacceptable levels of false negatives. An additional major challenge is building the targeted testing protocols that quickly provide data from complex integrated biological systems (e.g., complex in vitro cell systems, whole organisms) to evaluate the reliability of the toxicity signature. The idea is to provide the minimal level of chemical characterization to begin making more complex risk decisions. Currently, most data used for DNT hazard assessments are collected using historically accepted methods that are very resource intensive. The goal of this strategy is not to require full regulatory guideline testing for any chemicals prioritized for further testing. Needed are short-term in vivo methods that are more rapid and cost-efficient that conventional testing. Padilla's talk highlighted the use of zebrafish as an alternative species for this type of work.

Finally, the last level of testing in the Integrated Evaluation Strategy is reserved for those chemicals that need to be evaluated beyond screening and require a more thorough assessment to determine where in their life cycle the greatest potential for hazard and/or exposure exists. Systems modeling approaches can be applied to inform enhanced risk assessments for those chemicals suspected of nearing or exceeding the threshold of acceptable health or the involvement of highly susceptible exposed populations. Research at Level 4 will require a more complete understanding of the key elements of the toxicity pathways which underlie the phenotypic, cell-based data obtained using the assays described in this meeting, along with the demonstration of how data fits into adverse outcome pathways leading to developmental neurotoxicity. Initially, research at this level will cost more, but will result in reduced uncertainty in estimates of risk and improving the protection of human health.

\section{References}

Abbott, B. D., Held, G. A., Wood, C. R., et al. (1999). AhR, ARNT, and CYP1A1 mRNA quantitation in cultured human embryonic palates exposed to TCDD and comparison with mouse palate in vivo and in culture. Toxicol. Sci. 47, 62-75.

Albert, S. (2007). Projecting neurologic disease burden. Difficult but critical. Neurology 68, 322-323.

Altieri, L., Neri, C., Sacco, R., et al. (2011). Urinary p-cresol is elevated in small children with Autism Spectrum Disorder. Biomarkers 16, 252-260.

Amano, T., Richelson, E., and Nirenberg, M. (1972). Neurotransmitter synthesis by neuroblastoma clones (neuroblast differentiation-cell culture-choline acetyltransferase-acetylcholinesterase-tyrosine hydroxylase-axons-dendrites). Proc. Natl. Acad. Sci. U. S. A. 69, 258-263.

Andersen, M. E., Krewski, D. (2009). Toxicity testing in the $21^{\text {st }}$ century: bringing the vision to life. Toxicol. Sci. 107, 324330 .

Audouze, K. and Grandjean, P. (2011). Application of computational systems biology to explore environmental toxicity hazards. Environ. Health Perspect. 119, 1754-1759.

Bal-Price, A. K., Hogberg H. T., Buzanska, L., et al. (2010). In vitro developmental neurotoxicity (DNT) testing: relevant models and endpoints. Neurotoxicology 31, 545-554.

Bauman, M. L. and Kemper, T. L. (2005). Neuroanatomic observations of the brain in autism: a review and future directions. Int. J. Dev. Neurosci. 23, 183-187.

Bill, B. R., Petzold, A. M., Clark, K. J., et al. (2009). A primer for morpholino use in zebrafish. Zebrafish 6, 69-77.

Breier, J. M., Radio, N. M., Mundy, W. R., and Shafer, T.J. (2008). Development of a high-throughput screening assay for chemical effects on proliferation and viability of immortalized human neural progenitor cells. Toxicol. Sci. 105, 119-133.

Buzańska, L., Habich, A., Jurga, M., et al. (2005). Human cord blood-derived neural stem cell line--possible implementation in studying neurotoxicity. Toxicol. In Vitro 19, 991-999.

Buzanska, L., Sypecka, J., Nerini-Molteni, S., et al. (2009). A human stem cell-based model for identifying adverse effects of organic and inorganic chemicals on the developing nervous system. Stem Cells 27, 2591-2601.

Byers, R. and Lord, E. (1943). Late effects of lead poisoning on mental development. Am. J. Dis. Child 66, 471-494.

Campbell, D. B., D'Oronzio, R., Garbett, K., et al. (2007). Dis- 
ruption of cerebral cortex MET signaling in autism spectrum disorder. Ann. Neurol. 62, 243-250.

Campbell, D. B., Sutcliffe, J. S., Ebert, P. J., et al. (2006). A genetic variant that disrupts MET transcription is associated with autism. Proc. Natl. Acad. Sci. U. S. A. 103, 16834-16839.

Carayol, J., Sacco, R., Tores, F., et al. (2011). Converging evidence for an association of ATP2B2 allelic variants with autism in males. Biol. Psychiatry 70, 880-887.

Coecke, S., Goldberg, A. M., Allen, S., et al. (2007). Workgroup report: incorporating in vitro alternative methods for developmental neurotoxicity into international hazard and risk assessment strategies. Environ. Health Perspect. 115, 924-931.

Cory-Slechta, D. A., Thiruchelvam, M., Barlow, B. K., and Richfield, E. K. (2005). Developmental pesticide models of the Parkinson disease phenotype. Environ. Health Perspect. $113,1263-1270$.

Crofton, K. M., Mundy, W. R., Lein, P. J., et al. (2011). Developmental neurotoxicity Testing: recommendations for developing alternative methods for the screening and prioritization of chemicals. ALTEX 28, 9-15.

Donato, R., Miljan, E. A., Hines, S. J., et al. (2007). Differential development of neuronal physiological responsiveness in two human neural stem cell lines. BMC Neurosci. 8, 36.

Dorsey, E. R., Constantinescu, R., Thompson, J. P., et al . (2007). Projected number of people with Parkinson disease in the most populous nations, 2005 through 2030. Neurology 68, 384-386.

Elbaz, A., Bower, J. H., Maraganore, D. M., et al. (2002). Risk tables for Parkinsonism and Parkinson's disease. J. Clin. Epidemiol. 55, 25-31.

Environmental Working Group (2005). "Body burden - the pollution in newborns." http://www.ewg.org/reports/bodyburden2/ execsumm.php (last accessed March 30, 2012).

Eskenazi, B., Huen, K., Marks, A., et al. (2010). PON1 and neurodevelopment in children from the CHAMACOS study exposed to organophosphate pesticides in utero. Environ. Health Perspect. 118, 1775-1781.

Frimat, J. P., Sisnaiske, J., Subbiah, S., et al. (2010). The network formation assay: a spatially standardized neurite outgrowth analytical display for neurotoxicity screening. Lab Chip. 10, 701-709.

Fritsche, E., Cline, J. E., Nguyen, N. H., et al. (2005). Polychlorinated biphenyls disturb differentiation of normal human neural progenitor cells: clue for involvement of thyroid hormone receptors. Environ. Health Perspect. 113, 871-876.

Fritsche, E., Gassmann, K., and Schreiber, T. (2011). Neurospheres as a model for developmental neurotoxicity testing. Methods Mol. Biol. 758, 99-114.

Gaita, L., Manzi, B., Sacco, R., et al. (2010). Decreased serum arylesterase activity in Autism Spectrum Disorders. Psychiatry Res. 180, 105-113.

GAO - U.S. Government Accounting Office (2010). Chemical Regulation: Options exist to improve EPA's ability to assess health risks and manage its chemical review program. http:// www.gao.gov/new.items/d05458.pdf (last accessed March $30,2012)$.
Gassmann, K., Abel, J., Bothe, H., et al. (2010). Species-specific differential AhR-expression protects human neural progenitor cells against developmental neurotoxicity of PAHs. Environ. Health Perspect. 118, 1571-1577.

Geerts, D., Schilderink, N., Jorritsma, G., and Versteeg, R. (2003). The role of the MEIS homeobox genes in neuroblastoma. Cancer Lett. 197, 87-92.

Grandjean, P. and Landrigan, P. J. (2006). Developmental neurotoxicity of industrial chemicals. Lancet 368, 2167-2178.

Grandjean, P. (2008a). Late insights into early origins of disease. Basic Clin. Pharmacol. Toxicol. 102, 94-99.

Grandjean, P. (2008b). Seven deadly sins of environmental epidemiology and the virtues of precaution. Epidemiology 19, 158-162.

Grandjean, P. (2010). Even low-dose lead exposure is hazardous. Lancet 376, 855-856.

Grandjean, P., Eriksen, M. L., Ellegaard, O., and Wallin, J. A. (2011). The Matthew effect in environmental science publication: A bibliometric analysis of chemical substances in journal articles. Environ. Health 10, 96.

Greene, L. A., and Tischler, A. S. (1976). Establishment of a noradrenergic clonal line of rat adrenal pheochromocytoma cells which respond to nerve growth factor. Proc. Natl. Acad. Sci. U.S. A. 73, 2424-2428.

Grether, J. K., Rosen, N. J., Smith, K. S., and Croen, L. A. (2009). Investigation of shifts in autism reporting in the California Department of Developmental Services. J. Autism Dev. Disord. 39, 1412-1419.

Guo, S. (2009). Using zebrafish to assess the impact of drugs on neural development and function. Expert Opin. Drug Discov. 4, 715-726.

Gustafsson, H., Runesson, J., Lundqvist, J., et al. (2010). Neurofunctional endpoints assessed in human neuroblastoma SH-SY5Y cells for estimation of acute systemic toxicity. Toxicol. Appl. Pharmacol. 245, 191-202.

Hallmayer, J., Cleveland, S., Torres, A., et al. (2011). Genetic heritability and shared environmental factors among twin pairs with autism. Arch. Gen. Psychiatry 68, 1095-1102.

Harrill, J. A., Robinette, B. L., and Mundy, W. R. (2011). Use of high content image analysis to detect chemical-induced changes in synaptogenesis in vitro. Toxicol. In Vitro 25, 368-387.

Harrill, J. A., Freudenrich, T. M., Robinette, B. L., and Mundy, W.R. (2011). Comparative sensitivity of human and rat neural cultures to chemical-induced inhibition of neurite outgrowth. Toxicol. Appl. Pharmacol. 256, 268-280.

Hertz-Picciotto, I., Croen, L. A., Hansen, R., et al. (2006). The CHARGE study: an epidemiologic investigation of genetic and environmental factors contributing to autism. Environ. Health Perspect. 114, 1119-1125.

Higashijima, S. (2008). Transgenic zebrafish expressing fluorescent proteins in central nervous system neurons. Dev. Growth Differ. 50, 407-413.

Hogberg, H. T., Kinsner-Ovaskainen, A., Hartung, T., et al. (2009). Gene expression as a sensitive endpoint to evaluate cell differentiation and maturation of the developing central nervous system in primary cultures of rat cerebellar granule 
cells (CGCs) exposed to pesticides. Toxicol. Appl. Pharmacol. 235, 268-286.

Hogberg, H. T., Kinsner-Ovaskainen, A., Coecke, S., et al. (2010). mRNA expression is a relevant tool to identify developmental neurotoxicants using an in vitro approach. Toxicol. Sci. 113, 95-115.

Hogberg, H. T., Sobanski, T., Novellino, A., et al. (2011). Application of micro-electrode arrays (MEAs) as an emerging technology for developmental neurotoxicity: evaluation of domoic acid-induced effects in primary cultures of rat cortical neurons. Neurotoxicology 32, 158-168.

Judson, R. S., Mortensen, H. M., Shah, I., et al. (2012). Using pathway modules as targets for assay development in xenobiotic screening. Mol. Biosyst. 8, 531-542.

Julvez, J. and Grandjean, P. (2009). Neurodevelopmental toxicity risks due to occupational exposure to industrial chemicals during pregnancy. Ind. Health 47, 459-468.

Jurga, M., Markiewicz, I., Sarnowska, A., et al. (2006). Neurogenic potential of human umbilical cord blood: neural-like stem cells depend on previous long-term culture conditions. J. Neurosci.Res. 83, 627-637.

Jurga, M., Lipkowski, A. W., Lukomska, B., et al . (2009). Generation of functional neural artificial tissue from human umbilical cord blood stem cells. Tissue Eng. Part C Methods. 15, 365-372.

King, M. and Bearman, P. (2009). Diagnostic change and the increased prevalence of autism. Intl. J. Epidemiol. 38, 12241234.

Kohl, R. L., Perez-Polo, J. R., and Quay, W. B. (1980). Effect of methionine, glycine and serine on serine hydroxymethyltransferase activity in rat glioma and human neuroblastoma cells. J. Neurosci. Res. 5, 271-280.

Krey, J. and Dolmetsch, R. (2007). Molecular mechanisms of autism: a possible role for $\mathrm{Ca}^{2+}$ signaling. Curr. Opin. Neurobiol. 17, 112-119.

Landrigan, P. J. (2010). What causes autism? Exploring the environmental contribution. Curr. Opin. Pediatr. 22, 219-225.

Lucchini, R., Albini, E., Benedetti, L., et al. (2007). High prevalence of parkinsonian disorders associated to manganese exposure in the vicinities of ferroalloy industries. Am. J. Ind. Med. 50, 788-800.

Makris, S. L., Raffaele, K., Allen S., et al. (2009). A retrospective performance assessment of the developmental neurotoxicity study in support of OECD test guideline 426. Environ. Health Perspect. 117, 17-25.

Martin, M. T., Knudsen, T. B., Reif, D. M., et al. (2011). Predictive Model of rat reproductive toxicity from ToxCast high throughput screening. Biol. Reprod. 85, 327-339.

Moors, M., Cline, J. E., Abel, J., and Fritsche, E. (2007). ERKdependent and -independent pathways trigger human neural progenitor cell migration. Toxicol. Appl. Pharmacol. 221, 5767.

Moors, M., Rockel T. D., Abel, J., et al. (2009). Human neurospheres as three-dimensional cellular systems for developmental neurotoxicity testing. Environ. Health Perspect. 117, 1131-1138.
Mundy, W. R., Robinette, B., Radio, N. M., and Freudenrich, T. M. (2008). Protein biomarkers associated with growth and synaptogenesis in a cell culture model of neuronal development. Toxicology 249, 220-229.

Mundy, W. R., Radio, N. M., and Freudenrich, T. M. (2010). Neuronal models for evaluation of proliferation in vitro using high content screening. Toxicology 270, 121-130.

Nadeau, S. E. (1997). Parkinson's disease. J. Am. Geriatr. Soc. 45, 233-240.

Needham, L. L. P., Grandjean, B., Heinzow, P. J., et al. (2011). Partition of environmental chemicals between maternal and fetal blood and tissues. Environ. Sci. Technol.45, 1121-1126.

Novellino, A., Scelfo, B., Palosaari T., et al. (2011). Development of micro-electrode array based tests for neurotoxicity: assessment of interlaboratory reproducibility with neuroactive chemicals. Front. Neuroeng. 4, 4.

NRC - National Research Council (2007). Toxicity Testing in the $21^{\text {st }}$ Century: A Vision and a Strategy. Washington, DC: The National Academies Press.

OECD (2007). Test Guideline 426. OECD Guideline for Testing of Chemicals. Developmental Neurotoxicity Study. http://www. oecd.org/document/55/0,3343,en_2649_34377_2349687_1_1_ 1_1,00.html

Padilla, S. and MacPhail, R. C. (2011). Using zebrafish to assess developmental neurotoxicity. In R.C. Gupta (ed.). Reproductive and Developmental Toxicology. London: Academic Press, 179-192.

Palmieri, L., Papaleo, V., Porcelli, V., et al. (2010). Altered calcium homeostasis in autism-spectrum disorders: evidence from biochemical and genetic studies of the mitochondrial aspartate/glutamate carrier AGC1. Mol. Psychiatry 15, 38-52.

Persico, A. M. and Bourgeron, T. (2006). Searching for ways out of the autism maze: genetic, epigenetic and environmental clues. Trends Neurosci. 29, 349-358.

Pessah, I. N., Cherednichenko, G., and Lein, P. J. (2010). Minding the calcium store: Ryanodine receptor activation as a convergent mechanism of PCB toxicity. Pharmacol. Ther. 125, 260-285.

Pichery, C., Bellanger, M., Zmirou-Navier, D., et al. (2011). Childhood lead exposure in France: benefit estimation and partial cost-benefit analysis of lead hazard control. Environ. Health 10, 44.

Plunkett, L. M., Kaplan, A. M., and Becker, R. A. (2010). An enhanced tiered toxicity testing framework with triggers for assessing hazards and risks of commodity chemicals. Regul. Toxicol. Pharmacol. 58, 382-394.

Podrygajlo, G., Song, Y., Schlesinger, F., et al. (2010). Synaptic currents and transmitter responses in human NT2 neurons differentiated in aggregate culture. Neurosci. Lett. 468, 207210.

Radio, N. M., Breier, J. M., Shafer, T. J., and Mundy, W. R. (2008). Assessment of chemical effects on neurite outgrowth in PC12 cells using high content screening. Toxicol. Sci. 105, 106-18.

Radio, N. M., Freudenrich, T. M., Robinette, B. L., et al. (2010). Comparison of PC12 and cerebellar granule cell cultures for 
evaluating neurite outgrowth using high content analysis. Neurotoxicol. Teratol. 32, 25-35.

Rees, D. C., Francis, E. Z., and Kimmel, C. A. (1990). Scientific and regulatory issues relevant to assessing risk for developmental neurotoxicity: an overview. Neurotoxicol. Teratol. 12, 175-181.

Reynolds, C. P. and Pérez-Polo, J. R. (1975). Human neuroblastoma: Glial induced morphological differentiation. Neurosci Lett. 1, 91-97.

Richelson, E. (1973). Regulation of tyrosine hydroxylase activity in mouse neuroblastoma clone N1E-115. J. Neurochem. $21,1139-1145$.

Robinette, B. L., Harrill, J. A., Mundy, W. R., and Shafer, T. J. (2011). In vitro assessment of developmental neurotoxicity: use of microelectrode arrays to measure functional changes in neuronal network ontogeny. Front. Neuroeng. 4, 1.

Sarkanen, J. R., Nykky, J., Siikanen, J., et al. (2007). Cholesterol supports the retinoic acid-induced synaptic vesicle formation in differentiating human SH-SY5Y neuroblastoma cells. J. Neurochem. 102, 1941-1952.

Schreiber, T., Gassmann, K., Moors, M., et al. (2010). Polybrominated Diphenyl Ethers induce Developmental Neurotoxicity in a Human in vitro Model: Evidence for Endocrine Disruption. Environ. Health Perspect. 118, 572-578.

Scholz, D., Pöltl, D., and Genewsky, A. (2011). Rapid, complete and large-scale generation of post-mitotic neurons from the human LUHMES cell line. J. Neurochem. 119, 957-971.

Seiler, A., Baumann, W., Bicker, G., et al. (2007). The new German BMBF joint project on the development of predictive in vitro tests for developmental neurotoxicity testing. ALTEX $24,225$.

Seiler, A. and Spielmann, H. (2011). The validated embryonic stem cell test to predict embryotoxicity in vitro. Nat. Protoc. 6, 961-978.

Shafer, T. (2011). Assessment of 16 chemicals on proliferation and apoptosis in human neuroprogenitor cells using High Content Image Analysis (HCA). Abstract presented at DNT3 Conference. May 10-13, 2011. Ville Ponti Congress Centre, Varese, Italy.

Sheng, L., Ding, X., and Ferguson, M. (2010). Prenatal polycyclic aromatic hydrocarbon exposure leads to behavioral deficits and downregulation of receptor tyrosine kinase, MET. Toxicol. Sci. 118, 625-634.

Spielmann, H., Pohl, I., and Döring, B. (1997). The embryonic stem cell test, an in vitro embryotoxicity test using two permanent mouse cell lines: 3T3 fibroblasts and embryonic stem cells. In Vitro Mol. Toxicol. 10, 119-127.

Sun, W., Buzanska, L., and Domanska-Janik, K. (2005). Voltagesensitive and ligand-gated channels in differentiating neural stem-like cells derived from the nonhematopoietic fraction of human umbilical cord blood. Stem Cells 23, 931-945.

Tegenge, M. A., Rockel, T. D., Fritsche, E., and Bicker, G. (2010). Nitric oxide stimulates human neural progenitor cell migration via cGMP-mediated signal transduction. Cell. Mol. Life Sci. 68, 2089-2099.

Tsang, M. (2010). Zebrafish: A tool for chemical screens. Birth
Defects Research Part C: Embryo Today: Reviews 30, 185192.

Tao, T. and Peng, J. (2009). Liver development in zebrafish (Danio rerio). J. Genet. Genomics 36, 325-334.

US EPA - United States Environmental Protection Agency (1998). Health Effects Test Guidelines OPPTS 870.6300 Developmental Neurotoxicity Study. Washington DC, United States Environmental Protection Agency Office of Prevention, Pesticides and Toxic Substances. http://www.regulations. gov/\#!documentDetail;D=EPA-HQ-OPPT-2009-0156-0042

US EPA (1999). Guidance for identifying pesticide chemicals and other substances that have a common mechanism of toxicity. United States Environmental Protection Agency Office of Pesticides Program. http://www.epa.gov/fedrgstr/ EPA-PEST/1999/February/Day-05/6055.pdf

US EPA (2010a). TSCA New Chemicals Program: Chemical Categories. United States Environmental Protection Agency Office of Pollution Prevention and Toxics. http://www.epa. gov/oppt/newchems/pubs/npcchemicalcategories.pdf

US EPA (2010). Draft Framework for Incorporating $\mathrm{Hu}-$ man Epidemiologic \& Incident Data in Health Risk Assessment. United States Environmental Protection Agency.http://www.regulations.gov/\#!documentDetail;D=EPAHQ-OPP-2009-0851-0004

Vance, J. M., Ali, S., Bradley, W. G., et al. (2010). Gene-environment interactions in Parkinson's disease and other forms of Parkinsonism. Neurotoxicology 31, 598-602.

Willighagen, E. L., Jeliazkova, N., Hardy, B., et al. (2011). Computational toxicology using the OpenTox application programming interface and Bioclipse. BMC Res. Notes 4, 487.

Ylä-Outinen, L., Heikkilä, J., and Skottman, H. (2010). Human cell-based micro electrode array platform for studying neurotoxicity. Front. Neuroeng. 3. pii,111.

Zychowicz, M., Mehn, D., Ruiz, A., et al. (2011). Proliferation capacity of cord blood derived neural stem cell line on different micro-scale biofunctional domains. Acta Neurobiol. Exp. (Wars) $71,12-23$.

\section{Correspondence to:}

Anna Bal-Price, PhD

Validation of Alternative Methods Unit

Institute for Health and Consumer Protection

EC-Joint Research Centre

TP 580, Via E. Fermi 2749

21027 Ispra (VA), Italy

Phone: +390332 786018

e-mail: anna.price@jrc.ec.europa.eu 